\title{
Unmanned Aircraft Preliminary Design and Pneumatic Gauge
}

\author{
Xiujuan Liu ${ }^{\mathrm{a}}$, Guangxu Ren ${ }^{\mathrm{a}}$, Chunguang Wang ${ }^{\mathrm{a}}$ \\ Aviation University of Air Force, Changchun, China \\ 723704537@qq.com
}

Keywords: Unmanned aerial vehicle (uav); preliminary design; aerodynamic estimation

\begin{abstract}
The UAV found rapidly, it has the characteristics of manned aircraft do not have, such as cost, no risk of human life, and increasing people' s attention. Unmanned aerial vehicle aerodynamic design, it affects the entire airplane aerodynamic characteristics and flight performance, aircraft preliminary design the aerodynamic layout, ensure the success of the program. This article is intended primarily for unmanned aerial vehicle features preliminary selection of a number of programs ,including the wings, fuselage, tail, control parameter selection for main parts such as control surface, and the overall design of the program to be a preliminary discussion, unmanned aerial vehicles and providing a theoretical reference model plane enthusiasts, also design their own little UAV theory laid the foundation of the future.
\end{abstract}

\section{Introduction}

Propeller Unmanned aerial vehicle (uav) is a dynamic, can be controlled, can carry a variety of mission equipment and can be repeated use of unmanned aerial vehicle. Uav with a flexible, rapid response, the flight, low operation requirements, carrying more sensors, affect the real-time transmission, high-risk areas detection and high comprehensive benefit many advantages. Is a powerful supplement of satellite remote sensing and the traditional aerial remote sensing. Appear first in the world since 1913, since the autopilot, unmanned aerial vehicle (uav) is valued by more and more countries is developing rapidly.

\section{Unmanned Aircraft Aerodynamic Layout Design Train of Thought.}

Layout Forms. The existing unmanned aerial vehicle (uav) and in the world today are designing a new type of unmanned aerial vehicle (uav) is mainly divided into two categories: fixed wing and rotary, including fixed-wing unmanned aerial vehicle (uav) mainly adopts the following layout.

exhibition string than drones that general use or turbofan engine as the power piston engine, rear engine, design using larger aspect ratio, the speed is low. Its main advantage is rear engine can maximize the cabin of the space saving, easy to load the reconnaissance equipment, radar and other airborne mission equipment. The most prominent advantage is rather long battery life, combat radius more than $3000 \mathrm{~km}$. The unmanned aerial vehicle (uav) of several typical models with Israel's American heron, the explorers of the predator, South Africa and the United States global hawk.

small aspect ratio commonly used high-speed unmanned aerial vehicle (uav) turbojet engines or ramjet, the speed is higher, which can be applied to air defense fire stronger, but the task load is small, do not apply to the development of multipurpose aerial platform.

Choice of unmanned aerial vehicle (uav) configuration steps.1) close to the design requirements of aircraft information collection2) list all possible unmanned aircraft configuration3) to compare the advantages and disadvantages of the unmanned aircraft type.4) choose a layout model

Unmanned Aerial Vehicle (Uav) Mathematical Model. Used in aircraft preliminary design and detailed design stage of the model, on the other hand, should be detailed and complete as far as possible to consider the factors influence the selection of design scheme. Then the mathematical model can be considered as parametric model of the "real" aircraft design for the design of the product. According to the relationship of the aircraft performance and parameter can reflect the different aspects of its structure and function of group, consisting of a series of sub models, mainly include: 
(1)Geometric model, describe unmanned aerial vehicle (uav) the relationship between the parameters and the shape and size characteristics. In selected general plane layout and determination of plane geometry definition, some parameters such as shape, surface area, volume, and the wing, wing and fuselage sections, etc. The model data used for weight center of gravity, pneumatic and strength calculation, unmanned aircraft aerodynamic layout and internal layout, structure layout, and assembly with CNC process. Therefore, this model also become unified model.

(2)The weight model, a unified description of bearing system of uav geometry and structure, the structure and arrangement, plane loading condition and the relationship between the aircraft and parts weight.

(3)The aerodynamic model, describe the geometric characteristics of the unmanned aerial vehicle (uav) and the dynamic characteristics (in a variety of flight conditions of aerodynamic drag, lift and moment and force and moment coefficient) of the relationship between.

(4)Power plant model, and describe the various flight condition, engine size, layout and the relationship between the thrust and specific fuel consumption.

(5)The flight dynamics model, the description of the unmanned aerial vehicle (uav) flight performance and maneuver performance (speed range, range, rate of climb, ceiling, overload, etc.) and aerodynamic force, the weight of the unmanned aerial vehicle (uav) characteristics and the relationship between the power plant.

(6)Flight operation stability and control model, describe the geometric characteristics of the aircraft aerodynamic characteristics, weight characteristics (inertia), stability and maneuverability, and the relationship between the control system.

(7)Strength model, describe the aerodynamic load, weight, and the geometric characteristics of the unmanned aerial vehicle (uav) and the strength characteristics of bearing structure, plane stress levels and the relationship between the deformation size, widely used in the finite element method.

(8)Economy model, said the technical parameters of unmanned aerial vehicle (uav) and its design, the relationship between the production and use of cost.

\section{Important Parts Design Form.}

The Design Of The Wing. The major function of the wing is to generate lift, to support the aircraft in the air; Also has certain stability and control effect. General installation with ailerons and flaps on the wing. Manipulation of the ailerons can make the aircraft roll; Put down the flaps can make the wing lift increases. In addition, the wing can also install the engine, fuel tanks and landing gear. The wings have a variety of shapes, the number is different also. History has seen biplane, even appear too much. But modern aircraft are generally monoplane.

Wing Plane Shape. Low speed is generally straight wing airplane wings, due to its low speed aerodynamic characteristics is good, induced resistance is small, lift-to-drag ratio. Straight wing of the plane shape generally has two kinds of rectangular and trapezoidal.

In terms of pneumatic, rectangular wing root stall first, has intrinsic stall security features, and the load distribution of the wings are ideal. The trapezoidal wing soft wingtip stalling characteristics, when the root tip than choosing the right (approximate elliptical wing), the load distribution of the wings is also relatively moderate.

Section Shape of the Wing (Hereinafter Referred to as Airfoils). An airplane wing is parallel to the plane of symmetry plane section below When get, section called airfoil shape. Airfoil front-end Point, known as the leading edge; After the endpoint, known as the trailing edge. Leading edge and trailing edge attachment called chord, its length is called chord length or geometric chord length.

Early similar a bird's wing airfoil section of the aircraft. Above the wing facing the degree of bending is bigger, the surface is flat aerofoil said flat convex profile; Table facing the degree of bend than outward bending degree of the surface of airfoil say biconvex airfoil. Most modern aircraft wings at low speed using flat convex or biconvex airfoil. Up and down surface of chord symmetrical airfoil is called symmetric airfoils. Part of the high speed using symmetrical airfoil airplane wings. Supersonic aircraft requirements airfoils have sharp leading edge. Such as double arc airfoil, diamond 
airfoil. For balancing the aerodynamic characteristics of the speed range, the low supersonic aircraft still use small blunt symmetrical airfoil.

Wing Installation Location. The installation position of the wings on the fuselage usually has three kinds: The list of the wings, in the single wing and place the order with wings. These three forms, each has its own advantages and disadvantages.

The List Of The Wings. Advantages: 1) have good stability, on the Angle is small, the wing aerodynamic efficiency is higher. 2) the impact on its interior space is small, is advantageous to the fuselage arrangement of the internal loading. 3) wing body aerodynamic interference resistance smaller than single wing. 4) is connected to the fuselage than the simple single wing, fuselage and wing box structure can compensate each other, reduced the weight of the fuselage structure.

Disadvantages: 1) the gear such as on the wing, the height is too big, higher requirements for the gear stiffness. 2) if the single wing tricycle, after weight advantages than single wing and three point. 3) the abnormal landing, cannot provide protection on the fuselage.

In The Single Wing .Advantages: small wing body aerodynamic interference resistance.

Disadvantages: connected to the fuselage difficulties, had a greater influence on the internal space of the fuselage, unfavorable to the fuselage arrangement of the internal loading.

In The Single Wing. Advantages: 1) the abnormal landing, the wings on the fuselage protection. 2) the fuselage and wing box structure can compensate each other, reduced the weight of the fuselage structure. 3) impact on its interior space is small, is advantageous to the fuselage arrangement of the internal loading. 4) the gear installed on the wing, the height is small, the stiffness requirement is low, and the gear lighter weight.

Disadvantages: 1) poor stability, need large reverse Angle, reduce airfoil aerodynamic efficiency. 2) wing body aerodynamic interference resistance is bigger.

Lift Of The Wing Of The Estimate. Aircraft lift-to-drag features is the most important one of the original data of the plane, in such aspects as performance calculation, flight simulation is indispensable. Designs or in the process of aircraft design, especially in early design, the overall parameters such as aerodynamic layout is usually change, airfoil parameters such as has not been completely determined, thus calculate the accurate aerodynamic data is difficult. Engineering method is adopted to improve the aerodynamic estimation, usually for further calculation and analysis of the original parameters. In addition to foreign design of aircraft, unable to get accurate airfoil shape parameters, such as, also can only on the aerodynamic estimation to get the aerodynamic parameters.

The Tail Of The Preliminary Design. Tail includes vertical and horizontal tail wing. Tailplane by fixed level of surface and moving the elevator. Vertical tail, including fixed vertical fin and movable rudder. The major function of the tail is used to control the aircraft pitch and deflection, and ensure the plane can fly smoothly.

Flat Tail. Flat tail or flat tail, installed in the back, is mainly used to maintain the stability of the aircraft in flight and control the aircraft's flight. Is very similar to the internal structure of the tail and wings, usually made up of skeletal and skin, but their surface generally smaller size, the thickness of the thinner, there are some characteristics in structure form. In general, the tail by fixed tail plane and deflection of the elevator.

Vertical Tail. Vertical tail short tail, also called vertical tail, installed in the back, its function is similar to the tail, is also used to keep the stability of the aircraft in flight and flight attitude control of an airplane. Different vertical tail is around in the plane (yaw) direction of static stability, and control the plane in the direction of the left and right sides (yaw) movement. At the tail, vertical tail by fixed vertical fin and deflection of the rudder.

Tail Lift Estimate. Tail is divided into vertical and horizontal tail wing, and only the tail generate lift. Tail lift line slope, first of all, according to the single wing lifting line slope estimation method, calculate the single tail lifting line slope, again modification, mainly under the revised wash and block.

The Landing Gear Of The Preliminary Design. The landing gear design is a very important link in the design of unmanned aircraft. Landing gear's main function is to support and cushion, and to improve the aircraft's vertical direction and the vertical stress distribution, the gear taxiing, landing 
the plane took off ground and ground motion can bear larger load and slow movement, the impact, in order to enhance security.

Suitable for unmanned aerial vehicle (uav) landing gear form mainly has three before and after three point two, each have advantages and disadvantages.

The Plane Control Surfaces. Mainly includes the main control plane control surfaces and the auxiliary control surfaces.

Track Of Main Control Surface (Surface).Main control of the plane surface is also called the aircraft rudder surface, including installed in a horizontal tail after the trailing edge of the elevator, installed in a vertical tail rudder and installed in the trailing edge on the front of the (generally in the lateral) flap (as shown in figure 1-10).

Similar to the elevator and rudder, the aileron is installed in the wing tip of the outside of the trailing edge of a small piece of movable wing surface, is Lord of the aircraft rudder surface operation. About the pilot control of aileron deflection differential roll torque can make the aircraft roll motion. Aileron wingspan about $1 / 6$ of the whole wing span to about $1 / 5$ of the chord of the wing chord around $1 / 5$ to $1 / 4$.

Auxiliary Control Surfaces. Auxiliary control of the plane surface is refers to the installation of other active surface on the plane, such as: slats, flaps, spoiler (slow), adjustment and compensation pills, etc.

Through the front and back edge of the wings made movable, can change the profile of the wing of the camber and area, increase the lift, improve take-off and landing performance. This can increase the lift the activities of the wing surface is called rising device or flaps. General component is frontal flap, flap stitched wing trailing edge and leading edge flap. Airplane flaps usually used respectively or increase the comprehensive utilization of airfoil in arc curvature, increasing the wing area and delay flow separation of the three principles. Braking spoiler also known as "'," choke board "or" reduced rose board ", etc., these names reflect their function.

All The Machines, Pneumatic Estimation Method. Pneumatic estimate although wind tunnel test on the accuracy of accurate, nor as intuitive as computational fluid dynamics results show that the flow field, but it is a simple and effective engineering aerodynamic calculation method, can be quickly in the initial stages of the plane design basic aircraft aerodynamic data, used to guide the project design. Preliminary determine the plane layout form, get basic dimension of the aircraft, on the basis of in-depth study on solution.

The simplest computational estimation method in the selection of airfoil and related parts has been done in the related work, go here. The following introduced two methods of software simulation calculations, for your reference.

\section{ANSYSYS Application In Design Of Unmanned Aerial Vehicle (uav)}

ANSYSYS Software. ANSYS software for finite element theory is one of successful large-scale CAE software, has penetrated into various engineering fields. It can solve the statics problem, also can solve the dynamic problems; Can solve the problem of solid mechanics, can also solve the fluid mechanics problem; Can calculate the steady state thermal problems already, also can deal with the transient time response; ANSYS/FE - SAFE module can life calculation, especially for the engine and the the effect of rotating components fatigue calculation of the helicopter is particularly important. Through the analysis of ANSYS software technology that can help engineering design personnel before uav design or production prediction, simulation, the performance of the unmanned aerial vehicle (uav) calculation, so as to improve the quality of the performance of the aircraft.

ANSYSYS Analysis Process. Finite element analysis was carried out on the aircraft structure, general should pass load analysis, modeling, input material properties, boundary conditions, several main links such as the finite element method.

Digital DATCOM Program. 2 Digital DATCOM program is introduced. Digital DATCOM program is in DATCOM calculation on the basis of the development of manual calculation and program. With the test data of aircraft on the basis of empirical data fitting method to calculate the aerodynamic coefficient and derivative. Can directly get fuck needed for the stability analysis of 
aerodynamic derivatives, especially suitable for the initial design phase of the aerodynamic derivative calculation. The following for instance.

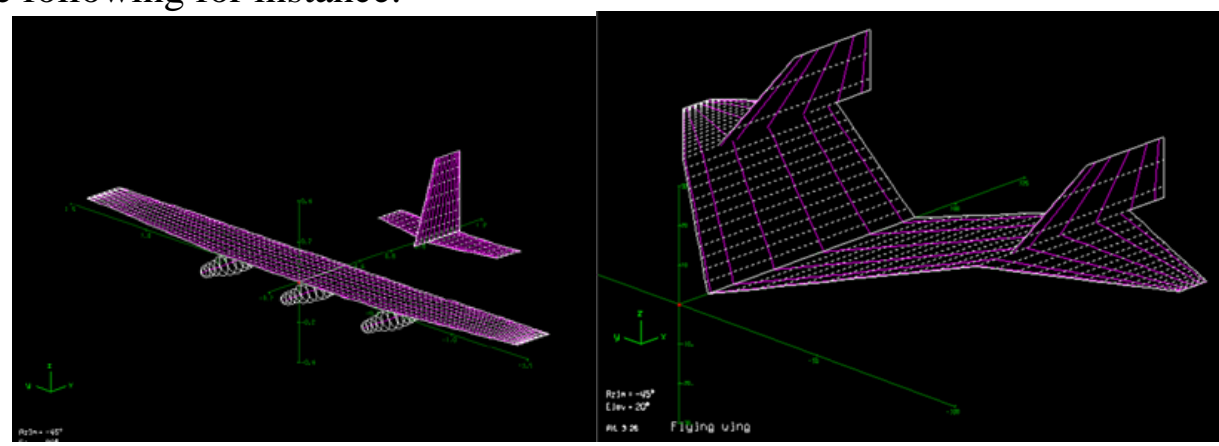

Fig.1 Digital DATCOM estimates

\section{The Main Function}

Digital DATCOM program main function is as follows:

1. The calculated output aerodynamic force and moment coefficient (basic).

2. Calculate the output static stability derivative (basic).

3. The output dynamic stability derivative calculation.

4. Calculate the flat end with the corresponding force and moment at ordinary times.

5. Calculate the force generated by the flaps, aileron and elevator torque.

In addition, also can cooperate in the process of estimating DWT (Digital Wing Tunnel) numerical wind Tunnel aerodynamic derivatives based on surface element method calculation software. Can be based on line of non sticky flow equation; The numerical method, the surface element method, the $\mathrm{C}$ language. Can estimate the lift, induced resistance, friction resistance, longitudinal/transverse lateral static/dynamic aerodynamic derivatives. The following for instance:

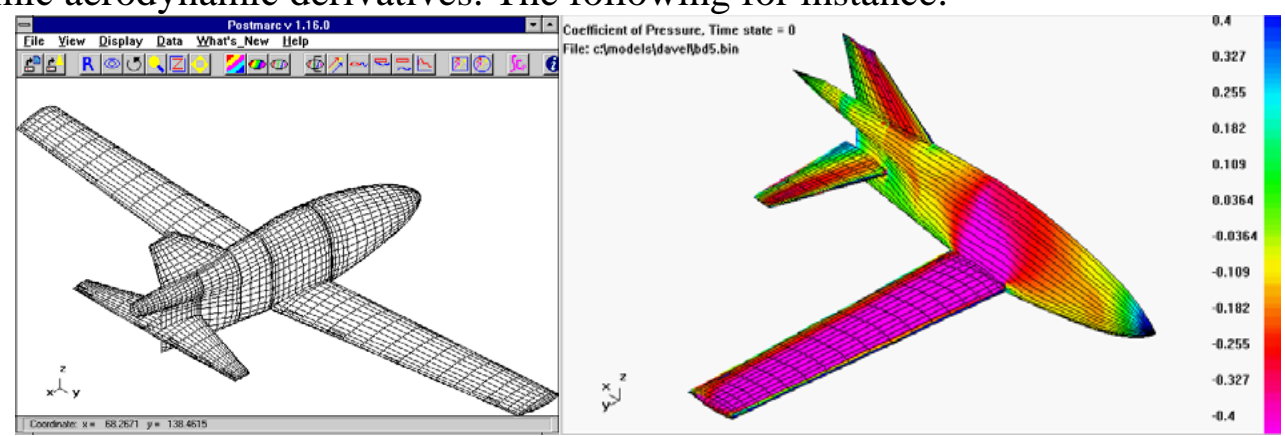

Fig.2 DWT estimate

\section{Summary}

Uav design needs to be dynamic, mathematics, aerodynamics, flight mechanics, pneumatic control, materials and technology, power system, mechanical design, structural mechanics, and other fields, such as application of science and the basic scientific knowledge together.

Aircraft in flight, except by the inertia force, but also affected by the aerodynamic force; And also as the flight status and flight conditions and aerodynamic force of different; Therefore, before using ANSYS software for finite element analysis, to analyze the aircraft aerodynamic load, determine the condition of power transmission way and the analysis, according to the different types of load at the same time, the standard and specification adopted by the finite element analysis is determined. Because a large number of unmanned aircraft thin-wall structure, can the simplification of analysis model in the ANSYS modeling; Aircraft parts of simple structure and can directly establish geometric model in ANSYS, generating finite element mesh, or directly to establish finite element model. The structure of complex, generally with CAD software such as UG, CATIA, etc.) to establish geometric model, through the interface program ANSYS, the CAD model into ANSYS software, after repair, regeneration into finite element model. 


\section{References}

[1] YanHengYuan, Aircraft aerodynamic characteristics analysis and estimate [M], northwestern polytechnical university press, 1990:

[2] LiuBin, Small electric uavs population parameter design method research Journal of Northwestern Polytechnical University[J].

[3]Shen GuangKan. Mechanical Simulation and Aerodynamic Analysis on a New Type of Wing Trailing Edge Variable Camber[J].

[4] ChenZheng Cai. Propeller slipstream - numerical wing interference effects. China Aerodynamics Research and Development Center .1980

[5] Chen TingNan. Aircraft flight performance and quality control [M], Beijing, national defense industry press 